\title{
Therapeutic Advances in Movement Disorders
}

\author{
Caroline M. Tanner ${ }^{1,2}$ (D) Jill L. Ostrem ${ }^{1}$ \\ Accepted: 3 December 2020 / Published online: 15 January 2021 \\ (C) This is a U.S. government work and not under copyright protection in the U.S.; foreign copyright protection may apply 2021
}

\section{Introduction}

The field of movement disorder management and therapeutics continues to evolve and change at a remarkable pace. Advances in our understanding of the underlying biology of diseases, new capabilities in genetics, and improved approaches to diagnosis, including biomarkers and imaging, provide clinicians today with a growing array of options to help improve the lives and outcomes of our patients (see Table 1). For this special edition of Neurotheraputics, we are fortunate to include twenty-seven contributions from experts leading the development and application of these new therapeutic options in movement disorders. Each can stand alone to serve as a comprehensive resource for a particular topic, but together they provide an outstanding and detailed synopsis of current therapeutics in the field. Many of the chapters focus on Parkinson's disease, but more than a third of the reviews highlight the remarkable transformation in therapeutic options for other conditions - perhaps most notably Huntington's disease and the tauopathies. Despite the progress highlighted, there still remains a great unmet need for improved treatments. The diseases we manage still lack neuroprotective or disease-modifying approaches, let alone cures. With an aging world population and prevalence increasing for the most common of these conditions [1], the urgency has never been greater to move beyond current therapeutics to relieve suffering and the burden of disease.

Caroline M. Tanner

caroline.tanner@ucsf.edu

1 Movement Disorder and Neuromodulation Center, Department of Neurology, Weill Institute for Neuroscience, University of California San Francisco, San Francisco, CA, USA

2 Parkinson's Disease Research, Education and Clinical Center, San Francisco Veterans Affairs Medical Care System, San Francisco, CA, USA

\section{Parkinson's Disease Therapeutics}

This issue of the Journal first focuses on nonsurgical approaches to treatment of the motor features of Parkinson's disease. With the ever growing therapeutic options available to treat $\mathrm{PD}$, determining the optimal therapeutic regimen for an individual patient has become increasingly complicated. Sy and Fernandez [2] review pharmacological strategies to consider when motor symptoms are sufficient to warrant treatment. There are many approved monotheraputic agents to choose from in early PD, requiring an individualized approach to optimize risk reduction while providing effective relief from symptoms. Next Aradi and Hauser [3] discuss the clinical features, epidemiology, and risk factors for the development of motor fluctuations and dyskinesia, which are commonly seen with short-acting medications as the disease advances. They also provide a helpful review of strategies to consider to maximize motor function and limit dyskinesiaa common clinical dilemma. Marras and colleagues [4] review current approaches to understanding PD subtypes including motor phenotype, age, nonmotor dominant symptoms, and genetic forms. There is much enthusiasm for these emerging data-driven approaches to better define PD subtypes based on clinical features and biomarkers with the hope of translating this into patient-specific therapeutic approaches. Schneider et al. [5] follow with a more focused description of emerging targeted therapeutics for specific genetic subtypes of parkinsonism. The first precision medicine approaches for treatment in PD may apply to GBA-associated and LRRK2-associated forms of the disease. An update on other emerging PD therapeutic approaches being explored that may influence disease progression is provided by Drs. Schwarzschild and Hung [6]. Novel immunotherapies and drugs targeting misfolded $\alpha$-synuclein, c-Abelson tyrosine kinase (cABl), Glucagon-like peptide-1 (GLP-I) agonists, calcium channel blockers, nicotine pathways, and reactive oxygen species are all under development and offer hope to the field despite the long history of multiple failed disease-modifying therapeutic compounds to date. Next, our current understanding about the 
Table 1 US FDA approvals for drugs and devices for movement disorders, 1/1/2016-11/30/2020

\begin{tabular}{|c|c|c|c|}
\hline Drug/device & Year & Disease & Indication \\
\hline
\end{tabular}

antipsychotic

Inverse agonist and antagonist at serotonin 5HT 2A receptors

ExAblate Neuro

Focused ultrasound

2016 Essential tremor (ET)

Neurostimulator (Abbott St Jude Medical Infinity DBS system and directional leads)

Amantadine extended release

(Gocovri)

NMDA receptor antagonist

Valbenazine (Ingrezza ${ }^{2,4,5}$ )

VMAT 2 inhibitor

Deutetrabenazine (Austedo ${ }^{2}$ )

VMAT 2 inhibitor

Safinamide (Xadago)

MAOB inhibitor

Neurostimulator (Vercise DBS System)

Incobotulinum toxin A $\left(\mathrm{Xeomin}^{2}\right)$ Inhibition of acetylcholine release Levodopa inhalation powder (Inbrija)

Dopamine precursor

Exablate Neuro

Fluorodopa (FDOPA) F18

Radioactive diagnostic agent

Istradefylline $^{1}$ (Nourianz)

Adenosine A2a receptor antagonist

Neurostimulator (Vercise PC and Gevia systems with Cartesia directional leads)

Opicapone (Ongentsys)

COMT inhibitor

Apomorphine hydrochloride (Kynmobi) 2020 Parkinson's disease (PD)

Non-ergoline dopamine agonist sublingual film

Neurostimulator (Abbott St. Jude Medical Infinity DBS System)

Neurostimulator (Medtronic Percept PC 2020 Parkinson's disease (PD); DBS System)
2020 Parkinson's disease (PD)

2016 Parkinson's disease (PD) Essential tremor (ET)

2017 Parkinson's disease (PD)

2017 Tardive dyskinesia (TD)

2017 Huntington's disease (HD); Tardive dyskinesia (TD)

2017 Parkinson's disease (PD)

2017 Parkinson's disease (PD)

2018 Adults with chronic sialorrhea

2018 Parkinson's disease (PD)

2018 Parkinson's disease (PD)

2019 Parkinsonian syndromes

2019 Parkinson's disease (PD)

2019 Parkinson's disease (PD)

2020 Parkinson's disease (PD)

Essential tremor (ET); Dystonia
In patients with ET whose tremors cannot be treated using medication. Patients must be at least 22 years old. The designated area in the brain responsible for the movement disorder symptoms (ventralis intermedius) must be identified and accessible for targeted thermal ablation by the ExAblate device.

Bilateral stimulation of the subthalamic nucleus (STN) as an adjunctive therapy to reduce some of the symptoms of advanced levodopa-responsive Parkinson's disease that are not adequately controlled by medications.

Unilateral or bilateral stimulation of the ventral intermediate nucleus (VIM) of the thalamus for the suppression of disabling upper extremity tremor in adult essential tremor patients whose tremor is not adequately controlled by medications and where the tremor constitutes a significant functional disability

The treatment of dyskinesia in patients with PD receiving levodopa therapy, with or without concomitant dopaminergic medications ${ }^{7}$

Adults with $\mathrm{TD}^{7}$

Chorea associated with HD; TD in adults

Adjunctive treatment to levodopa/carbidopa in patients with PD experiencing off episodes

Indicated for use in bilateral stimulation of the subthalamic nucleus (STN) as an adjunctive therapy in reducing some of the symptoms of moderate to advanced levodopa-responsive PD that are not adequately controlled with medication

Chronic sialorrhea ${ }^{8}$

The intermittent treatment of OFF episodes in patients with PD treated with carbidopa/levodopa

Expanded to include unilateral thalamotomy for patients with tremor-dominant PD with medication-refractory tremor

Radioactive diagnostic agent for use in PET to visualize dopaminergic nerve terminals in the striatum for evaluation of adults with suspected Parkinsonism, as adjunct to other diagnostic evaluations.

Adjunctive treatment to levodopalcarbidopa in adult patients with PD experiencing “off” episodes

Indicated for use in bilateral stimulation of the subthalamic nucleus (STN) as an adjunctive therapy in reducing some of the symptoms of moderate to advanced levodopa-responsive PD that are not adequately controlled with medication

Adjunctive treatment to levodopa/carbidopa in adult patients with PD experiencing "off" episodes

Acute intermittent treatment of "off" episodes in people with PD

Expanded lead placement options to include bilateral GPi to reduce some of the motor symptoms of levodopa-responsive PD not controlled by medication

PD: Bilateral stimulation of the GPi or STN is indicated for adjunctive therapy in reducing some of the symptoms in individuals with levodopa-responsive PD of at least 4 years' duration that are not adequately controlled with medication, including motor 
Table 1 (continued)

\begin{tabular}{ll}
\hline Drug/device & Year Disease \\
\hline & $\begin{array}{l}\text { Indication } \\
\text { complications of recent onset (from } 4 \text { months to } 3 \text { years) or motor } \\
\text { Tremor: Unilateral thalamic stimulation of the ventral intermediate } \\
\text { nucleus (VIM) is indicated for the suppression of tremor in the upper } \\
\text { extremity. The system is intended for use in patients who are } \\
\text { diagnosed with ET tremor or parkinsonian tremor not adequately } \\
\text { controlled by medications and where the tremor constitutes a } \\
\text { significant functional disability } \\
\text { Dystonia: Unilateral or bilateral stimulation of the GPi or STN is } \\
\text { indicated as an aid in the management of chronic, intractable (drug } \\
\text { refractory) primary dystonia, including generalized and/or segmental } \\
\text { dystonia, hemidystonia, and cervical dystonia (torticollis), in patients } \\
7 \text { years of age or above }\end{array}$ \\
\hline
\end{tabular}

New drugs, new class III devices, new drug formulations and expanded use indications for movement disorders approved for use in the USA between January 2016 and November 2020 are presented in the table

$5 \mathrm{HT}=5$ hydroxytryptamine; VMAT $=$ vesicular monoamine transporter; $\mathrm{MAOB}=$ monoamine oxidase $\mathrm{B} ; \mathrm{DBS}=$ deep brain stimulation; $\mathrm{COMT}=$ catechol-O-methyltransferase

FDA CDER review designations: ${ }^{1}$ First in class— drugs with a new and unique mechanism for treating a medical condition; ${ }^{2}$ Orphan — drugs approved for small populations of patients with rare diseases. Regulatory methods to expedite development and approval of novel drugs: ${ }^{3}$ Fast track - drugs that can treat unmet medical needs; ${ }^{4}$ Breakthrough — a drug with preliminary clinical evidence demonstrating that it may result in substantial improvement on at least one clinically significant endpoint over other available therapies; ${ }^{5}$ Priority Review — is given if the drug could potentially provide a significant advance in medical care; ${ }^{6}$ Accelerated approval — early approval based on markers that predict a reasonable benefit, with more testing to confirm clinical benefit after approval. Other: ${ }^{7}$ First indication; ${ }^{8}$ Expanded use

Source: www.fda.gov

understudied, yet likely important, influence of the gut microbiome in PD is highlighted by Brown and Goldman including strategies now under study to modulate the microbiome to improve the effects of PD medications absorbed through the gut [7]. Schootemeijer et al. [8] provide a wonderful summary of the role of aerobic exercise in PD. They present evidence including a meta-analysis, which supports the notion that aerobic exercise has general health benefits, improves physical fitness, and attenuates motor symptoms in PD. Dosing of exercise is important and compliance issues remain a challenging barrier. Complementary and alternative medicine approaches have never been more popular in the PD community, including acupuncture, tai qi, yoga, meditation, and cannabis. Deuel and Seeberger [9] review for us the latest in clinical trials evaluating the effectiveness of these complementary treatments, which have a newly appreciated role in management of PD. The tremendous benefit of palliative care methods in Parkinson's spectrum disorders is thoughtfully reviewed by Katz [10]. Rather than reserving palliative approaches for late-stage disease, she emphasizes the value of this multidisciplinary approach for patients and caregivers at any stage of disease.

The PD field has increasingly recognized the enormous impact nonmotor PD symptoms have on quality of life and the historically limited therapeutic options available to manage them. We have included four articles that each address current and emerging management of aspects of nonmotor symptoms that greatly increase the burden of disease in PD. The broad clinical spectrum of autonomic dysfunction is discussed by Pfeiffer et al. [11]. Importantly, if these symptoms are identified, they can often be managed with the many available non-PD specific approaches. Sleep disorders are common in PD, but under-diagnosed, as Zuzuarregui and During report in their comprehensive review [12]. They cover the current understanding and approaches to treatment of REM sleep behavior disorders (RBD), restless legs syndrome (RLS), obstructive sleep apnea (OSA) and circadian rhythm dysfunction, noting that treating these disorders can significantly improve quality of life. Perhaps one of the greatest unmet needs in the field of PD is the prevention and management of cognitive decline and dementia. Uc et al. describe strategies to diagnose and treat this very difficult nonmotor aspect of PD [13]. Weintraub discusses the increasingly complicated topic of management of psychiatric disorders in PD, another area with significant impact on quality of life [14]. Psychiatric disorders are still under-recognized and undertreated in PD, and even though psychotropic medications are commonly used, we lack randomized controlled trials demonstrating efficacy and tolerability specific to PD.

Rounding out the chapters on Parkinson's disease therapeutics are two that focus on surgical approaches. Surgical treatment approaches have transformed the management of moderate to advanced PD care and, besides the discovery of levodopa, represent one of the greatest therapeutic 
breakthroughs for the field. Sharma et al. review the latest in DBS and lesioning approaches in PD including indications, patient selection, and management strategies and help us to appreciate how this rapidly changing area is becoming more complex with application of multiple DBS systems, improved technology, and leveraging a more sophisticated understanding of PD pathophysiology [15]. Buttery and Barker end the PD section on a hopeful note, with a wonderful review of where we are now with gene- and cell-based therapies [16], many of which are in ongoing high-profile clinical trials. They highlight the great promise for these approaches, some of which may allow for slowing progression of the disease or medication reduction, without the need for implanted hardware.

\section{Therapeutics in Other Movement Disorders}

The next section of this issue of the Journal includes manuscripts that highlight current therapeutics for movement disorders other than Parkinson's disease. Treatment of other forms of neurodegenerative parkinsonism has long been fraught with challenges. Many of these disorders are relatively rare, and clinical syndromes may be varied despite similar pathology, making both diagnosis and treatment challenging. An example is the 4 repeat tauopathies, where clinical syndromes may be predominantly motor or behavioral, or any combination. Following an excellent pragmatic overview of currently available options for symptomatic treatment in the four-repeat tauopathies, VandeVrede and colleagues provide hope for disease-modifying therapies in their comprehensive review of clinical trials [17]. Treatment of multiple system atrophy (MSA), an $\alpha$-synucleinopathy, provides similar challenges in clinical heterogeneity. Burns and McFarland provide a useful guide to diagnosis and treatment of the core motor symptoms of the several clinical syndromes of MSA, importantly emphasizing the value of a multidisciplinary care team in providing effective therapy that integrates nonpharmacologic management and drug treatment. Their review of current trials to identify disease-modifying therapies provides hope for more effective interventions in the future [18]. Hopfner and Deuschl review the two-axis Movement Disorder Society (MDS) classification for essential tremor syndrome, providing a foundational approach to determining therapeutics in this common but heterogeneous movement disorder [19]. They provide a comprehensive overview of nonpharmacologic, pharmacologic, and surgical treatments, and a useful framework for determining a patient-specific therapeutic plan. Dystonia comprises a heterogeneous group of syndromes with diverse etiologies. Therapeutic approaches to dystonia are equally broad. Bledsoe et al. provide a masterful overview of the many existing and emerging therapies for dystonia, ranging from pathogenesis-targeted therapies to symptomatic treatments with drugs or botulinum toxin, surgical therapies, noninvasive stimulation, and rehabilitation [20]. They highlight the need for an individualized approach to each patient and provide a helpful starting point for clinicians. Next, Dash and Mestre discuss the rapidly developing therapeutic options for Huntington's disease including symptomatic treatments and treatments that target specific pathways in the HD biology [21]. Novel huntingtin protein-lowering therapies using antisense oligonucleotides, RNA interference, small-molecule splicing modulators, and zinc-finger protein transcription factor approaches are likely to bring the first ever diseasemodifying therapy to patients with HD. They also address the need for specific biomarkers, improved rating scales, and development of other tools to identify disease progression necessary to move therapeutic advances for the disease forward. Then we turn to the latest medication strategies and emerging therapies for ataxia, which are highlighted by Perlman [22]. While there still remain no FDA-approved therapies for ataxia, a hopeful pipeline of disease-modifying therapies is discussed. In the meantime, multiple drugs are available for symptomatic management of coexisting symptoms, and there are many medications that are used to treat ataxia off-label. Pena and Caviness then review a physiology-based treatment of myoclonus, a symptom that is often resistant to treatment. Myoclonus is currently best managed by identifying the underlying etiology and using medications that best address the neurophysiological subtype [23]. Tourette syndrome is a heterogeneous neurobehavioral disorder, which develops in childhood, presenting with motor and phonic tics but frequently accompanied by behavioral comorbidities that require a tailored management approach. Billnitzer and Jankovic highlight current behavioral, pharmacologic, and surgical treatment strategies and also review therapeutics in development for Tourette syndrome [24]. In recent years, new strategies have emerged for management of tardive syndromes (TS), which are highlighted in an excellent review by Factor [25]. Covered are the pathogenesis, clinical features, and epidemiology, followed by a comprehensive discussion of use of the new vesicular monoamine transporter type 2 inhibitors recently approved by the FDA. Other medications, botulinum toxin and surgical therapies can also contribute in the management of this common syndrome, but hopefully, future efforts will focus on the need for prevention and/or reversal of this iatrogenic movement disorder. Deputy and Tilton review common management strategies for pediatric movement disorders not covered in previous chapters, largely focusing on disorders of tone and cerebral palsy but also highlighting disorders representative of these heterogeneous disorders, including glucose transporter type 1 (Glut-1) deficiency, Sydenham's chorea and neurodegeneration with brain iron accumulation 
(NBIA) [26]. They provide a useful discussion of the unique aspects of management of children with movement disorders.

\section{Other Novel Factors and Approach to Therapeutics}

The two final reviews in this special edition address and highlight other novel factors that influence today's clinical trials. Vaswani et al. describe the critical issue of overcoming barriers to Parkinson's disease trial participation by using novel trial designs for recruitment and ways to increase participation and diversity [27]. While they focus on Parkinson's disease, their insights are generally applicable to all movement disorders. Lastly, Artusi et al. provide a fascinating look at current mobile health technologies, their use in clinical trials today, and the great potential for these approaches to revolutionize the field of movement disorders clinical care and clinical trials in the near future [28].

\section{Conclusion/Final Words}

Overall, the reviews in this issue provide a hopeful outlook for movement disorder therapeutics in the next decade. Improved symptomatic therapies, disease-modifying interventions, and approaches to disease prevention are in reach for many disorders. We wish to thank all the authors for their outstanding contributions to this issue, especially during the challenging times of the COVID-19 pandemic and its impact. We also thank the editorial team at Neurotherapeutics and, in particular, Maral Mouradian and Linda Powell, for their advice and support. We are humbled and honored to serve as coeditors.

Required Author Forms Disclosure forms provided by the authors are available with the online version of this article.

Supplementary Information The online version contains supplementary material available at https://doi.org/10.1007/s13311-020-00988-2.

\section{References}

1. Dorsey ER, Sherer T, Okun MS, Bloem BR. The Emerging Evidence of the Parkinson Pandemic. Journal of Parkinson's disease. 2018;8(s1):S3-S8.

2. Sy MAC, Fernandez HH. Pharmacological Treatment of Early Motor Manifestations of Parkinson Disease (PD). Neurotherapeutics : the journal of the American Society for Experimental NeuroTherapeutics. 2020.
3. Aradi SD, Hauser RA. Medical Management and Prevention of Motor Complications in Parkinson's Disease. Neurotherapeutics : the journal of the American Society for Experimental NeuroTherapeutics. 2020.

4. Marras C, Chaudhuri KR, Titova N, Mestre TA. Therapy of Parkinson's Disease Subtypes. Neurotherapeutics : the journal of the American Society for Experimental NeuroTherapeutics. 2020.

5. Schneider SA, Hizli B, Alcalay RN. Emerging Targeted Therapeutics for Genetic Subtypes of Parkinsonism. Neurotherapeutics : the journal of the American Society for Experimental NeuroTherapeutics. 2020. .

6. Schwarzschild M HA. Approaches to disease modification for Parkinson's disease: Clinical trails and lessions learned. Neurotherapeutics : the journal of the American Society for Experimental NeuroTherapeutics. 2020.

7. Brown EG, Goldman SM. Modulation of the Microbiome in Parkinson's Disease: Diet, Drug, Stool Transplant, and Beyond. Neurotherapeutics : the journal of the American Society for Experimental NeuroTherapeutics. 2020.

8. Schootemeijer S, van der Kolk NM, Bloem BR, de Vries NM. Current Perspectives on Aerobic Exercise in People with Parkinson's Disease. Neurotherapeutics : the journal of the American Society for Experimental NeuroTherapeutics. 2020.

9. Deuel LM, Seeberger LC. Complementary Therapies in Parkinson Disease: a Review of Acupuncture, Tai Chi, Qi Gong, Yoga, and Cannabis. Neurotherapeutics : the journal of the American Society for Experimental NeuroTherapeutics. 2020.

10. Katz M. Palliative care for Parkinson's spectrum disorders: An emerging approach. Neurotherapeutics : the journal of the American Society for Experimental NeuroTherapeutics. 2020.

11. Pfeiffer RF. Autonomic Dysfunction in Parkinson's Disease. Neurotherapeutics : the journal of the American Society for Experimental NeuroTherapeutics. 2020.

12. Zuzuarregui JRP, During EH. Sleep Issues in Parkinson's Disease and Their Management. Neurotherapeutics : the journal of the American Society for Experimental NeuroTherapeutics. 2020.

13. Uc E Z, Aldridge, Narayanan, Anderson. Approach to cognitive impairment in Parkinson's disease neurotheraputics. 2020.

14. Weintraub D. Management of psychiatric disorders in Parkinson's disease : Neurotherapeutics - Movement Disorders Therapeutics. Neurotherapeutics : the journal of the American Society for Experimental NeuroTherapeutics. 2020.

15. Sharma VD, Patel M, Miocinovic S. Surgical Treatment of Parkinson's Disease: Devices and Lesion Approaches. Neurotherapeutics : the journal of the American Society for Experimental NeuroTherapeutics. 2020.

16. Buttery PC, Barker RA. Gene and Cell-Based Therapies for Parkinson's Disease: Where Are We? Neurotherapeutics : the journal of the American Society for Experimental NeuroTherapeutics. 2020.

17. VandeVrede L, Ljubenkov PA, Rojas JC, Welch AE, Boxer AL. Four-Repeat Tauopathies: Current Management and Future Treatments. Neurotherapeutics : the journal of the American Society for Experimental NeuroTherapeutics. 2020.

18. Burns MR, McFarland NR. Current Management and Emerging Therapies in Multiple System Atrophy. Neurotherapeutics : the journal of the American Society for Experimental NeuroTherapeutics. 2020.

19. Hopfner F, Deuschl G. Managing Essential Tremor. Neurotherapeutics : the journal of the American Society for Experimental NeuroTherapeutics. 2020.

20. Bledsoe IO, Viser AC, San Luciano M. Treatment of Dystonia: Medications, Neurotoxins, Neuromodulation, and Rehabilitation. Neurotherapeutics : the journal of the American Society for Experimental NeuroTherapeutics. 2020. 
21. Dash D, Mestre TA. Therapeutic Update on Huntington's Disease: Symptomatic Treatments and Emerging Disease-Modifying Therapies. Neurotherapeutics : the journal of the American Society for Experimental NeuroTherapeutics. 2020.

22. Perlman SL. Update on the Treatment of Ataxia: Medication and Emerging Therapies. Neurotherapeutics : the journal of the American Society for Experimental NeuroTherapeutics. 2020.

23. Pena AB, Caviness JN. Physiology-Based Treatment of Myoclonus. Neurotherapeutics : the journal of the American Society for Experimental NeuroTherapeutics. 2020.

24. Billnitzer A, Jankovic J. Current Management of Tics and Tourette Syndrome: Behavioral, Pharmacologic, and Surgical Treatments. Neurotherapeutics : the journal of the American Society for Experimental NeuroTherapeutics. 2020.

25. Factor SA. Management of Tardive Syndrome: Medications and Surgical Treatments. Neurotherapeutics : the journal of the American Society for Experimental NeuroTherapeutics. 2020.
26. Deputy SR, Tilton AH. Treatment of disorders of tone and other considerations of pediatric movement disorders. Neurotherapeutics : the journal of the American Society for Experimental NeuroTherapeutics. 2020.

27. Vaswani PA, Tropea TF, Dahodwala N. Overcoming Barriers to Parkinson Disease Trial Participation: Increasing Diversity and Novel Designs for Recruitment and Retention. Neurotherapeutics : the journal of the American Society for Experimental NeuroTherapeutics. 2020.

28. Artusi CA, Imbalzano G, Sturchio A, Pilotto A, Montanaro E, Padovani A, et al. Implementation of Mobile Health Technologies in Clinical Trials of Movement Disorders: Underutilized Potential. Neurotherapeutics : the journal of the American Society for Experimental NeuroTherapeutics. 2020.

Publisher's Note Springer Nature remains neutral with regard to jurisdictional claims in published maps and institutional affiliations. 